\title{
Editorial
}

\section{Development of neuropsychiatry services worldwide}

Arguably Neuropsychiatry is one of the oldest psychiatric disciplines. It has even been suggested that psychiatry itself evolved from recognition and care of patients with neuropsychiatric disorders (1). Yet, it is ironic that neuropsychiatry is still seen in the 21st century as an evolving discipline which is attempting to carve its own identity amongst a number of established psychiatric subspecialties.

So, what are the hurdles on the path of appropriate provision of neuropsychiatric services and how can they be overcome? The problem does not lie in the recognition of prevalence of neuropsychiatric comorbidity in neurological patients, its impact on quality of life of neurological patients or the burden it places on patients and their carers. There is consistent and ever-growing evidence in a number of neurological conditions and from primary care as well as specialist settings that highlights the impact of neuropsychiatric problems on neurological illnesses and their outcomes (2-4). Need and demand for neuropsychiatric input have been quantified and service models to meet such a need have been proposed (4). So why is such a vital need still not met?

Divergence of neurology and psychiatry as separate medical disciplines from the 19th century onwards has often been blamed for gradually reducing neuropsychiatric/biological emphasis in psychiatric practice. As a consequence, neuropsychiatry is often misunderstood and has been seen sometimes as highly esoteric and exotic, something away from mainstream clinical practice (5). Unfortunately, this impression still persists despite growing evidence of neuropsychiatric need. The divergence of the two disciplines also had an impact on the training that neurologists received in psychiatry and psychiatrists in neurology in a number of countries. In addition, there were shortcomings in neuropsychiatric training for neurologists and psychiatrists (5). This meant that the clinicians under-recognised neuropsychiatric needs and if they did recognise them, they often did not know how to meet those needs (6).
The review by Arambepola, Rickards and Cavanna in the present issue looks at the issue of definition of neuropsychiatry, historical issues including boundaries of neurology and psychiatry, evolving roles of neuropsychiatrists and challenges for future neuropsychiatrists. They highlight the lack of consensus in defining the discipline and its boundaries, even though the nature and range of the essential skill set required by neuropsychiatrists is not in dispute.

Different definitions and interpretations of the core neuropsychiatric territory could be damaging the development of essential neuropsychiatric services globally. This needs to be resolved urgently. While the peripheral boundaries of neuropsychiatry are blurred and arguably could shift with time, the core territory of neuropsychiatry remains relatively stable and that is where neuropsychiatric services of the future must focus first.

In their review, Arambepola, Rickards and Cavanna suggest that there is a clear need for a specialised field of neuropsychiatry in fulfilling the complex needs of core neuropsychiatric patients. They highlight the consequences of the narrow focus of paradigms of psychiatry and neurology and thus the need for a broader neuropsychiatric perspective in a number of patients.

There are existing drivers for the development of neuropsychiatric services. Psychological, psychiatric and behavioural needs of patients with neurological conditions have been recognised in the national service framework for long-term conditions in the UK, heralding a shift away from the narrow medical models of the past (7). National Institute for Clinical Excellence (NICE) guidelines for a number of neurological conditions in the UK mention the need for psychological and neuropsychiatric support $(8,9)$. While these principles are universally applicable they often are not emphasised enough in these documents and recommendations could be more specific and strong. Greater neuropsychiatric involvement 
in development of such guidelines and frameworks, nationally and internationally, is urgently required.

Development of a neuropsychiatric core curriculum and a well-defined neuropsychiatric training pathway are essential for the development of neuropsychiatric services worldwide. This has been achieved successfully in the United States where there is now a board examination and certification in neuropsychiatry and behavioural neurology (10). However, broader neurological training for psychiatrists and psychiatric training for neurologists is equally important as the whole range of neuropsychiatric need cannot be met by specialist neuropsychiatrists. Indeed, it is often suggested that milder psychological comorbidities in neurological patients, such as depression, could very well be identified and treated by neurologists (11).

The challenges in evolution of neuropsychiatric services also include lack of adequate understanding about neuropsychiatry amongst commissioners and managers of neurosciences and mental health services. This is not surprising given differing definitions of neuropsychiatry and lack of certainty about its boundaries. This is combined with the lack of adequate national and international drivers and ambiguities in some places about who should 'own' neuropsychiatry services (neurosciences or mental health). Hence, guidelines are urgently needed for the current and future commissioners and providers of neuropsychiatric services.

There is evidence of growing interest in neuropsychiatry and anecdotal evidence of growth in neuropsychiatry services worldwide, which is encouraging. An unpublished survey of psychiatric trainees in the UK conducted by the Section of Neuropsychiatry of The Royal College of Psychiatrists showed that over three quarters of trainees wanted training in neuropsychiatry, while looking for a career in the whole range of psychiatric subspecialties. This energy and strong interest need to be harnessed.

Despite this, in reality, there remains a wide gap between current service provision and what could be considered adequate even for the core neuropsychiatric territory (4). While the discipline of neuropsychiatry is described to be in the middle of a global renaissance, the challenges for adequate service development remain immense. To usher in a new era of comprehensive neuropsychiatric care, a concerted effort is required worldwide.

\section{Niruj Agrawal \\ Department of Neuropsychiatry, St George's Hospital, London, UK}

\section{References}

1. Robinson RG. The neuropsychiatry in the age of subspecialisation. Eur J Psychiatry 2006;20:61-64.

2. Global Parkinson's Disease Survey Steering Committee. Factors impacting on quality of life in Parkinson's disease: results from an international survey. Mov Disord 2002; 17:60-67.

3. Corrigan JD, Whiteneck G, Mellick D. Perceived needs following traumatic brain injury. J Head Trauma Rehabil 2004;19:205-216.

4. Agrawal N, Fleminger S, Ring H, Deb S. Neuropsychiatry in the UK: planning the service provision for the $21 \mathrm{st}$ century. Psychiatr Bull 2008;32:303-306.

5. Mitchell AJ, Agrawal N. Training in neuropsychiatry: is it time to reintegrate into mainstream psychiatry? Psychiatr Bull 2005;29:361-364.

6. Fink P, Hansen MS, Sondergaard L, Frydenberg M. Mental illness in new neurological patients. J Neurol Neurosurg Psychiatr 2003;74:817-819.

7. Agrawal N, Mitchell AJ. The national service framework for long term conditions. BMJ 2005;330:1280-1281.

8. URL http://www.nice.org.uk/CG035 [accessed on 9 July 2012]

9. URL http://www.nice.org.uk/cg137 [accessed on 9 July 2012]

10. Silver JM. Behavioral Neurology and Neuropsychiatry Is a Subspecialty. J Neuropsychiatry Clin Neurosci 2006;18:146-148.

11. Agrawal N, Rickards H. Detection and treatment of depression in neurological disorders. J Neurol Neurosurg Psychiatr 2011;82:828-829. 